\title{
Thermal corrections to Rényi entropies for conformal field theories
}

\section{Christopher P. Herzog and Jun Nian}

C. N. Yang Institute for Theoretical Physics, Department of Physics and Astronomy, Stony Brook University, Stony Brook, NY 11794, U.S.A.

E-mail: cpherzog@insti.physics.sunysb.edu, Jun.Nian@stonybrook.edu

ABstract: We compute thermal corrections to Rényi entropies of $d$ dimensional conformal field theories on spheres. Consider the $n$th Rényi entropy for a cap of opening angle $2 \theta$ on $S^{d-1}$. From a Boltzmann sum decomposition and the operator-state correspondence, the leading correction is related to a certain two-point correlation function of the operator (not equal to the identity) with smallest scaling dimension. More specifically, via a conformal map, the correction can be expressed in terms of the two-point function on a certain conical space with opening angle $2 \pi n$. In the case of free conformal field theories, this two-point function can be computed explicitly using the method of images. We perform the computation for the conformally coupled scalar. From the $n \rightarrow 1$ limit of our results, we extract the leading thermal correction to the entanglement entropy, reproducing results of arXiv:1407.1358.

Keywords: Conformal and W Symmetry, Thermal Field Theory, Holography and condensed matter physics (AdS/CMT)

ARXIV EPRINT: 1411.6505 


\section{Contents}

1 Introduction 1

2 Analytical calculation $\quad 4$

3 The free case $\quad 5$

$\begin{array}{ll}3.1 & \text { The free scalar }\end{array}$

$\begin{array}{ll}3.2 & \text { Odd dimension and contour integrals } \\ \end{array}$

4 Thermal corrections to entanglement entropy 10

5 Numerical check $\quad 12$

6 Discussion 13

$\begin{array}{ll}\text { A Two-point functions in } d=(4+1) \text { dimensions } & 15\end{array}$

B Examples of thermal corrections to Rényi entropies 16

\section{Introduction}

Entanglement entropy plays an increasingly important role in different branches of physics. Proposed as a useful measure of the quantum entanglement of a system with its environment, entanglement entropy now features in discussions of black hole physics [1, 2], renormalization group flow [3, 4], and quantum phase transitions [5, 6]. A closely related set of quantities are the Rényi entropies. In this paper, our modest goal is to obtain thermal corrections to Rényi entropies for conformal field theory (CFT).

We adopt the conventional definition of entanglement and Rényi entropy in this paper. Suppose the space on which the theory is defined can be divided into a piece $A$ and its complement $\bar{A}=B$, and correspondingly the Hilbert space factorizes into a tensor product. The density matrix over the whole Hilbert space is $\rho$; then the reduced density matrix is defined as

$$
\rho_{A} \equiv \operatorname{tr}_{B} \rho
$$

The entanglement entropy is the von Neumann entropy of $\rho_{A}$,

$$
S_{E} \equiv-\operatorname{tr} \rho_{A} \log \rho_{A},
$$

while the Rényi entropies are defined to be

$$
S_{n} \equiv \frac{1}{1-n} \log \operatorname{tr}\left(\rho_{A}\right)^{n} .
$$


Assuming a satisfactory analytic continuation of $S_{n}$ can be obtained, the entanglement entropy can alternately be expressed as a limit of the Rényi entropies:

$$
\lim _{n \rightarrow 1} S_{n}=S_{E}
$$

To apply the results to a real system, it would be useful to know the thermal corrections to the entanglement entropy $S_{E}$ and the Rényi entropy $S_{n}$. Ref. [7] found universal thermal corrections to both $S_{E}$ and $S_{n}$ for a CFT on $S^{1} \times S^{1}$. The CFT is assumed to be gapped by having placed it on a spatial circle of circumference $L$, while the circumference of the second circle is the inverse temperature $\beta$. The results are

$$
\begin{aligned}
& \delta S_{n} \equiv S_{n}(T)-S_{n}(0)=\frac{g}{1-n}\left[\frac{1}{n^{2 \Delta-1}} \frac{\sin ^{2 \Delta}\left(\frac{\pi \ell}{L}\right)}{\sin ^{2 \Delta}\left(\frac{\pi \ell}{n L}\right)}-n\right] e^{-2 \pi \beta \Delta / L}+o\left(e^{-2 \pi \beta \Delta / L}\right), \\
& \delta S_{E} \equiv S_{E}(T)-S_{E}(0)=2 g \Delta\left[1-\frac{\pi \ell}{L} \cot \left(\frac{\pi \ell}{L}\right)\right] e^{-2 \pi \beta \Delta / L}+o\left(e^{-2 \pi \beta \Delta / L}\right)
\end{aligned}
$$

where $\Delta$ is the smallest scaling dimension among the set of operators not equal to the identity and $g$ is their degeneracy. The quantity $\ell$ is the length of the interval $A{ }^{1}$

To generalize the results of ref. [7] to higher dimensions, ref. [14] considered thermal corrections to the entanglement entropy $S_{E}$ on spheres. More precisely, a conformal field theory on $S^{1} \times S^{d-1}$ is considered in ref. [14], where the radius of $S^{1}$ and the one of $S^{d-1}$ are $\beta / 2 \pi$ and $R$ respectively. The region $A \subset S^{d-1}$ is chosen to be a cap with polar angle $\theta<\theta_{0}$. Then the thermal correction to the entanglement entropy $S_{E}$ is

$$
\delta S_{E}=g \Delta I_{d}\left(\theta_{0}\right) e^{-\beta \Delta / R}+o\left(e^{-\beta \Delta / R}\right)
$$

where

$$
I_{d}\left(\theta_{0}\right) \equiv 2 \pi \frac{\operatorname{Vol}\left(S^{d-2}\right)}{\operatorname{Vol}\left(S^{d-1}\right)} \int_{0}^{\theta_{0}} d \theta \frac{\cos \theta-\cos \theta_{0}}{\sin \theta_{0}} \sin ^{d-2} \theta
$$

The result (1.7) was derived employing a conformal transformation to hyperbolic space. The map can be used to specify the form of the so-called modular Hamiltonian $H_{M}=$ $-\log \rho_{A}$ almost completely. As ref. [14] noticed, the naive version nof the map sometimes misses boundary terms. In particular, when operators of conformal dimension $\Delta=d-2$ exist, the result (1.7) may be corrected. For a conformally coupled scalar, $\phi^{2}$ has dimension $d-2$ and a boundary term in the modular Hamiltonian means that the correction to entanglement entropy is given not by eq. (1.7) but by eq. (1.7) where $I_{d}\left(\theta_{0}\right)$ is replaced by $I_{d-2}\left(\theta_{0}\right)$. (For free fermions, however, there is no such shift [15].)

A natural question is how to calculate the thermal corrections to the Rényi entropy in higher dimensions. We would like to address this issue in the paper. Our main results

\footnotetext{
${ }^{1}$ The fact that $S_{E}(T)-S_{E}(0) \sim e^{-2 \pi \beta \Delta} / L$ is Boltzmann suppressed was conjectured more generally for gapped theories in ref. [8]. That $S_{E}(T)-S_{E}(0)$ might have a universal form for $1+1$ dimensional CFTs was suggested by the specific examples worked out in refs. [9-12]. See ref. [13] for higher order temperature corrections when the first excited state is created by the stress tensor.
} 
are the following. The thermal correction to the Rényi entropy for a cap-like region with opening angle $2 \theta_{0}$ on the sphere $S^{d-1}$ in $\mathbb{R} \times S^{d-1}$ is given by

$$
\delta S_{n}=\frac{n}{1-n}\left(\frac{\left\langle\psi(z) \psi\left(z^{\prime}\right)\right\rangle_{n}}{\left\langle\psi(z) \psi\left(z^{\prime}\right)\right\rangle_{1}}-1\right) e^{-\beta E_{\psi}}+o\left(e^{-\beta E_{\psi}}\right)
$$

where $\psi(z)$ is the operator that creates the first excited state of the CFT and $E_{\psi}$ is its energy. ${ }^{2}$ If we assume that $\psi(z)$ has scaling dimension $\Delta$, then we know further that $E_{\psi}=\Delta / R$. The two point function $\left\langle\psi(z) \psi\left(z^{\prime}\right)\right\rangle_{n}$ is evaluated on an $n$-fold cover of $\mathbb{R} \times S^{d-1}$ that is branched over the cap of opening angle $2 \theta_{0}$. Note the result (1.9) and the steps leading up to it are essentially identical to a calculation and intermediate result derived in ref. [7] in $1+1$ dimensions. The difference is that in $1+1$ dimensions, the two-point function $\left\langle\psi(z) \psi\left(z^{\prime}\right)\right\rangle_{n}$ can be evaluated for a general CFT through an appropriate conformal transformation, while in higher dimensions we only know how to evaluate $\left\langle\psi(z) \psi\left(z^{\prime}\right)\right\rangle_{n}$ in some special cases.

In the case of free fields (and perhaps more generally) it makes sense to map this $n$-fold cover of the sphere to $\mathcal{C}_{n} \times \mathbb{R}^{d-2}$ where $\mathcal{C}_{n}$ is a two dimensional cone of opening angle $2 \pi n$. In the case of a free theory, the two-point function $\left\langle\psi(y) \psi\left(y^{\prime}\right)\right\rangle_{1 / m}$, where $y$, $y^{\prime} \in \mathcal{C}_{n} \times \mathbb{R}^{d-2}$, can be evaluated by the method of images on a cone of opening angle $2 \pi / m$ and then analytically continued to integer values of $1 / \mathrm{m}$. Ref. [16] made successful use of this trick to calculate a limit of the mutual information for conformally coupled scalars. We will use this same trick to look at thermal corrections to Rényi entropies for these scalars. Taking the $n \rightarrow 1$ limit, we find complete agreement with entanglement entropy corrections computed in ref. [14], i.e. eq. (1.7) with $I_{d}(\theta)$ replaced by $I_{d-2}(\theta)$. (The method of images can also be used to study free fermions, but we leave such a calculation for future work [15].) We verify the Rényi entropy corrections numerically by putting the system on a lattice.

We should emphasize that the replica trick and the numerical lattice methods we employ to calculate the entanglement and Rényi entropies are not sensitive to the boundary term issues that occur in the hyperbolic space mapping employed in ref. [14]. The numerical methods rely on computing a two-point function on a space without a boundary, for which there is no boundary term we could possibly add. For the replica trick, one could conceivably insert an operator along the branching surface. The fact that the numerical and replica trick calculations agree, however, appears to rule out such a possibility. In short, these boundary term issues make eq. (1.7) not the universal result it could be, but do not really concern us in the present work.

The paper is organized as follows. In section 2, we derive the result (1.9) analytically. In section 3 , we describe the conformal map to $\mathcal{C}_{n} \times \mathbb{R}^{d-2}$ and then work out the specific case of the conformally coupled scalar field. Section 4 computes thermal corrections to entanglement entropy by considering the $n \rightarrow 1$ limit of the Rényi entropy corrections of section 3. The corrections agree with the results presented in ref. [14]. Section 5 provides a numerical check of the Rényi entropy corrections. We conclude in section 6 with a

\footnotetext{
${ }^{2}$ For simplicity, we have assumed that the first excited state is unique. For a degenerate first excited state, see the next section.
} 
summary, discussion of related problems, and proposals for future research. Appendix A provides details of a contour integral calculation of the scalar Green's function in $d=5$ dimensions, while appendix B summarizes examples of thermal Rényi entropy corrections for the scalar for small values of $n$ and $d$.

\section{Analytical calculation}

We start with the thermal density matrix:

$$
\rho=\frac{|0\rangle\left\langle 0\left|+\sum_{i}\right| \psi_{i}\right\rangle\left\langle\psi_{i}\right| e^{-\beta E_{\psi}}+\cdots}{1+g e^{-\beta E_{\psi}}+\cdots},
$$

where $|0\rangle$ stands for the ground state, while $\left|\psi_{i}\right\rangle(i=1, \cdots, g)$ denote the first excited states. For a conformal field theory on $\mathbb{R} \times S^{d-1}$,

$$
E_{\psi}=\frac{\Delta}{R},
$$

where $\Delta$ is the scaling dimension of the operators that create the states $\left|\psi_{i}\right\rangle$, and $R$ is the radius of the sphere. From this expression one can calculate that

$$
\begin{aligned}
\operatorname{tr}\left(\rho_{A}\right)^{n} & =\left(\frac{1}{1+g e^{-\beta E_{\psi}}+\cdots}\right)^{n} \cdot \operatorname{tr}\left[\operatorname{tr}_{B}\left(|0\rangle\left\langle 0\left|+\sum_{i}\right| \psi_{i}\right\rangle\left\langle\psi_{i}\right| e^{-\beta E_{\psi}}+\cdots\right)\right]^{n} \\
& =\operatorname{tr}\left(\operatorname{tr}_{B}|0\rangle\langle 0|\right)^{n} \cdot\left[1+\left(\frac{\operatorname{tr}\left[\operatorname{tr}_{B} \sum_{i}\left|\psi_{i}\right\rangle\left\langle\psi_{i}\right|\left(\operatorname{tr}_{B}|0\rangle\langle 0|\right)^{n-1}\right]}{\operatorname{tr}\left(\operatorname{tr}_{B}|0\rangle\langle 0|\right)^{n}}-g\right) n e^{-\beta E_{\psi}}+\cdots\right] .
\end{aligned}
$$

Then the thermal correction to the Rényi entropy is

$$
\begin{aligned}
\delta S_{n} & \equiv S_{n}(T)-S_{n}(0) \\
& =\frac{n}{1-n} \sum_{i}\left(\frac{\operatorname{tr}\left[\operatorname{tr}_{B}\left|\psi_{i}\right\rangle\left\langle\psi_{i}\right|\left(\operatorname{tr}_{B}|0\rangle\langle 0|\right)^{n-1}\right]}{\operatorname{tr}\left(\operatorname{tr}_{B}|0\rangle\langle 0|\right)^{n}}-1\right) e^{-\beta E_{\psi}}+o\left(e^{-\beta E_{\psi}}\right) .
\end{aligned}
$$

Hence, the crucial step is to evaluate the expression

$$
\frac{\operatorname{tr}\left[\operatorname{tr}_{B}\left|\psi_{i}\right\rangle\left\langle\psi_{i}\right|\left(\operatorname{tr}_{B}|0\rangle\langle 0|\right)^{n-1}\right]}{\operatorname{tr}\left(\operatorname{tr}_{B}|0\rangle\langle 0|\right)^{n}}=\frac{\left\langle\psi_{i}(z) \psi_{i}\left(z^{\prime}\right)\right\rangle_{n}}{\left\langle\psi_{i}(z) \psi_{i}\left(z^{\prime}\right)\right\rangle_{1}},
$$

which, using the operator-state correspondence, can be viewed as a two-point function on the $n$-fold covering of the space $\mathbb{R} \times S^{d-1}$. (Let $z^{\mu}$ be our coordinate system on $\mathbb{R} \times S^{d-1}$.) The $n$ copies are glued sequentially together along $A$. Let $\tau$ be the time coordinate. To create the excited state, we insert the operator $\psi_{i}$ in the far Euclidean past $\tau^{\prime}=-i \infty$ of one of the copies of $\mathbb{R} \times S^{d-1}$. Similarly, $\left\langle\psi_{i}\right|$ is created by inserting $\psi_{i}$ in the far future $\tau=i \infty$ of the same copy. The two-point function $\left\langle\psi_{i}(z) \psi_{i}\left(z^{\prime}\right)\right\rangle_{1}$ is needed in the denominator in order to insure that $\left\langle\psi_{i}\right|$ has the correct normalization relative to $\left|\psi_{i}\right\rangle$. 
Our most general result is then

$$
\delta S_{n}=\frac{n}{1-n} \sum_{i}\left(\frac{\left\langle\psi_{i}(z) \psi_{i}\left(z^{\prime}\right)\right\rangle_{n}}{\left\langle\psi_{i}(z) \psi_{i}\left(z^{\prime}\right)\right\rangle_{1}}-1\right) e^{-\beta E_{\psi}}+o\left(e^{-\beta E_{\psi}}\right) .
$$

Following from the analytic continuation formula (1.4), the thermal correction to the entanglement entropy can be determined via

$$
\delta S_{E}=\lim _{n \rightarrow 1} \delta S_{n}
$$

\section{The free case}

Evaluating the two-point function $\left\langle\psi_{i}(z) \psi_{i}\left(z^{\prime}\right)\right\rangle_{n}$ on an $n$-sheeted copy of $\mathbb{R} \times S^{d-1}$ is not simple for $n>1$. Using a trick of ref. [16], we can evaluate $\left\langle\psi_{i}(z) \psi_{i}\left(z^{\prime}\right)\right\rangle_{n}$ for free CFTs. The trick is to perform a conformal transformation that relates this two-point function to a two-point function on a certain conical space where the method of images can be employed. As interactions spoil the linearity of the theory and hence the principle of superposition, we expect this method will fail for interacting CFTs.

It is convenient to break the conformal transformation into two pieces. First, it is well known that $\mathbb{R} \times S^{d-1}$ is conformally related to Minkowski space (see the appendix of ref. [17]):

$$
\begin{aligned}
d s^{2} & =-d t^{2}+d r^{2}+r^{2} d \Omega^{2} \\
& =\Omega^{2}\left(-d \tau^{2}+d \theta^{2}+\sin ^{2} \theta d \Omega^{2}\right),
\end{aligned}
$$

where

$$
\begin{aligned}
t \pm r & =\tan \left(\frac{\tau \pm \theta}{2}\right) \\
\Omega & =\frac{1}{2} \sec \left(\frac{\tau+\theta}{2}\right) \sec \left(\frac{\tau-\theta}{2}\right),
\end{aligned}
$$

and $d \Omega^{2}$ is a line element on a unit $S^{d-2}$ sphere. Note that the surface $t=0$ gets mapped to $\tau=0$, and on this surface $r=\tan (\theta / 2)$. Thus a cap on the sphere (at $\tau=0$ ) of opening angle $2 \theta$ is transformed into a ball inside $\mathbb{R}^{d-1}$ (at $t=0$ ) of radius $r=\tan (\theta / 2$ ). This coordinate transformation takes the operator insertion points $\tau= \pm i \infty$ in the far past and far future (with $\theta=0$ ) to $t= \pm i$ (and $r=0$ ).

Then we should employ the special conformal transformation

$$
\begin{aligned}
y^{\mu} & =\frac{x^{\mu}-b^{\mu} x^{2}}{1-2 b \cdot x+b^{2} x^{2}} \\
d s^{2} & =d y^{\mu} d y^{\nu} \delta_{\mu \nu}=\frac{1}{\left(1-2 b \cdot x+b^{2} x^{2}\right)^{2}} d x^{\mu} d x^{\nu} \delta_{\mu \nu} .
\end{aligned}
$$

We let $x^{0}$ and $y^{0}$ correspond to Euclidean times. We consider a sphere of radius $r$ in the remaining $d-1$ dimensions, centered about the origin. If we set $b^{1}=1 / r$ and the rest of the $b^{\mu}=0$, this coordinate transformation will take a point on the sphere to infinity, specifically 
the point $x^{\mu}=(0, r, 0, \ldots, 0)$. The rest of the sphere will get mapped to a hyperplane with $y^{1}=-r / 2$. We can think of the total geometry as a cone in the $\left(y^{0}, y^{1}\right)$ coordinates formed by gluing $n$-spaces together, successively, along the half plane $y^{0}=0$ and $y^{1}<-r / 2$. Let us introduce polar coordinates $(\rho, \phi)$ on the cone currently parametrized by $\left(y^{0}, y^{1}\right)$. The tip of the cone $\left(y^{0}, y^{1}\right)=(0,-r / 2)$ will correspond to $\rho=0$. The insertion points $( \pm 1,0, \ldots, 0)$ for the operator $\psi_{i}$ get mapped to $( \pm 1,-1 / r, 0, \ldots, 0) /\left(1+1 / r^{2}\right)$. In polar coordinates, the insertion points of the $\psi_{i}$ are at $(r / 2, \pm \theta)$. By a further rescaling and rotation, we can put the insertion points at $(1,2 \theta, \overrightarrow{0})$ and $(1,0, \overrightarrow{0})$.

For primary fields $\psi_{i}(x)$, the effect of a conformal transformation on the ratio (2.5) is particulary simple. Let us focus on one of the $\psi_{i}=\psi$ and assume that it is a primary scalar field. We have

$$
\begin{aligned}
& \psi(x)=\left(\frac{1}{2} \sec \left(\frac{\tau+\theta}{2}\right) \sec \left(\frac{\tau-\theta}{2}\right)\right)^{-\Delta} \psi(z), \\
& \psi(y)=\left(1-2 b \cdot x+b^{2} x^{2}\right)^{\Delta} \psi(x)
\end{aligned}
$$

We are interested in computing

$$
\frac{\left\langle\psi(z) \psi\left(z^{\prime}\right)\right\rangle_{n}}{\left\langle\psi(z) \psi\left(z^{\prime}\right)\right\rangle_{1}}
$$

where the subscript $n$ indicates this $n$-fold covering of the sphere, glued along the boundary of $A$. In the ratio, the conformal factors relating the $z$ coordinates to the $x$ coordinates and the $x$ coordinates to the $y$ coordinates will drop out. All we need pay attention to is where $y$ and $y^{\prime}$ are in the cone of opening angle $2 \pi n$, which we have already done. For non-scalar and non-primary operators, the transformation rules are more involved.

For free CFTs, $\left\langle\psi(y) \psi\left(y^{\prime}\right)\right\rangle_{1 / m}$ can be evaluated for $m=1,2,3, \ldots$ by the method of images. For $n=1 / m$, the conical space has opening angle $2 \pi / m$. Let us assume we know the two-point function on $\mathbb{R}^{d}:\left\langle\psi\left(y_{1}\right) \psi\left(y_{2}\right)\right\rangle_{1}=f\left(y_{12}^{2}\right)$. Using the parametrization $y=(\rho, \theta, \vec{r})$, the square of the distance between the points is

$$
y_{12}^{2}=\rho_{1}^{2}+\rho_{2}^{2}-2 \rho_{1} \rho_{2} \cos \left(\theta_{12}\right)+\left(\vec{r}_{12}\right)^{2} .
$$

By the method of images,

$$
\left\langle\psi\left(y_{1}\right) \psi\left(y_{2}\right)\right\rangle_{1 / m}=\sum_{k=0}^{m-1} f\left(\rho_{1}^{2}+\rho_{2}^{2}-2 \rho_{1} \rho_{2} \cos \left(\theta_{12}+2 \pi k / m\right)+\left(\vec{r}_{12}\right)^{2}\right) .
$$

We are interested in two particular insertion points $y=(1,2 \theta, \overrightarrow{0})$ and $y^{\prime}=(1,0, \overrightarrow{0})$, for which the two point function reduces to

$$
\left\langle\psi(y) \psi\left(y^{\prime}\right)\right\rangle_{1 / m}=\sum_{k=0}^{m-1} f(2-2 \cos (2 \theta+2 \pi k / m)) .
$$

Once we have obtained an analytic expression for all $m$, we can then evaluate it for integer $n=1 / m$. 


\subsection{The free scalar}

We now specialize to the case of a free scalar, for which the scaling form of the Green's function in flat Euclidean space is $f\left(y^{2}\right)=y^{2-d}$. Our strategy will be to take advantage of recurrence relations that relate the Green's function in $d$ dimensions to $d+2$ dimensions. Let us define

$$
G_{(n, d)}^{B}(2 \theta) \equiv\left\langle\psi(y) \psi\left(y^{\prime}\right)\right\rangle_{n}
$$

We need to compute the sum

$$
G_{(1 / m, d)}^{B}(2 \theta)=\left\langle\psi(y) \psi\left(y^{\prime}\right)\right\rangle_{1 / m}=\sum_{k=0}^{m-1} \frac{1}{\left[2-2 \cos \left(2 \theta+\frac{2 \pi k}{m}\right)\right]^{\frac{d-2}{2}}} .
$$

As can be straightforwardly checked, this sum obeys the recurrence relation

$$
G_{(1 / m, d+2)}^{B}(\theta)=\frac{1}{(d-2)(d-1)}\left[\left(\frac{d-2}{2}\right)^{2}+\frac{\partial^{2}}{\partial \theta^{2}}\right] G_{(1 / m, d)}^{B}(\theta) .
$$

The most efficient computation strategy we found is to compute $G_{(n, d)}^{B}$ for $d=3$ and $d=4$ and then to use the recurrence relation to compute the two point function in $d>4$. (In $d=2$, the scalar is not gapped and there will be additional entanglement entropy associated with the degenerate ground state.)

To compute $G_{(n, 4)}^{B}$, and more generally $G_{(n, d)}^{B}$ when $d$ is even, we introduce the generalized sum

$$
f_{a}(m, \theta, z, \bar{z}) \equiv \sum_{k=0}^{m-1} \frac{1}{\left|z-e^{i(\theta+2 \pi k / m)}\right|^{2 a}},
$$

With this definition, we have the restriction that

$$
\lim _{z, \bar{z} \rightarrow 1} f_{(d-2) / 2}(m, \theta, z, \bar{z})=G_{(1 / m, d)}^{B}(\theta)
$$

and the recurrence relation

$$
\frac{\partial^{2} f_{a}}{\partial z \partial \bar{z}}=a^{2} f_{a+1}(m, \theta, z, \bar{z})
$$

In the case $d=4$, we find that

$$
f_{1}(m, \theta, z, \bar{z})=\frac{m}{|z|^{2}-1}\left[\frac{1}{1-z^{-m} e^{i m \theta}}+\frac{1}{1-\bar{z}^{-m} e^{-i m \theta}}-1\right] .
$$

The two-point function can be obtained from eq. (3.18) by taking the limit $z, \bar{z} \rightarrow 1$ :

$$
G_{(n, 4)}^{B}(\theta)=\lim _{z, \bar{z} \rightarrow 1} f_{1}\left(\frac{1}{n}, \theta, z, \bar{z}\right)=\frac{1}{n^{2}\left[2-2 \cos \left(\frac{\theta}{n}\right)\right]} .
$$


For $d=6$ dimensions the two-point function can be obtained by taking the $z, \bar{z} \rightarrow 1$ of $f_{2}(m, \theta, z, \bar{z})$ :

$$
G_{(n, 6)}^{B}(\theta)=\lim _{z, \bar{z} \rightarrow 1} f_{2}\left(\frac{1}{n}, \theta, z, \bar{z}\right)=\frac{1+\frac{2}{n^{2}}+\left(\frac{1}{n^{2}}-1\right) \cos \left(\frac{\theta}{n}\right)}{3 n^{2}\left[2-2 \cos \left(\frac{\theta}{n}\right)\right]^{2}} .
$$

Applying the recurrence relation (3.14) to the four dimensional result (3.19) yields the same answer. It is straightforward to calculate the Green's function in even $d>6$.

For $d=3$, we do not have as elegant expression for general $n$. Through a contour integral argument we will now discuss, for $n=1,2$, and 3 we obtain

$$
\begin{aligned}
G_{(1,3)}^{B}(\theta) & =\frac{1}{2 \sin \frac{\theta}{2}}, \\
G_{(2,3)}^{B}(\theta) & =\frac{1-\frac{\theta}{2 \pi}}{2 \sin \frac{\theta}{2}}, \\
G_{(3,3)}^{B}(\theta) & =\frac{1}{2 \sin \frac{\theta}{2}}\left[1-\frac{2}{\sqrt{3}} \sin \frac{\theta}{6}\right] .
\end{aligned}
$$

More general expressions for $G_{(n, d)}^{B}(\theta)$ with $d$ odd can be found in the next section. Tables of thermal Rényi entropy corrections $\delta S_{n}$ for some small $d$ and $n$ are in appendix B.

\subsection{Odd dimension and contour integrals}

Following ref. [16], for $d$ an odd integer we express the Green's function in terms of an integral and evaluate it using the Cauchy residue theorem:

$$
\begin{aligned}
G_{(1 / m, d)}^{B}(\theta) & =\sum_{k=0}^{m-1} \frac{1}{\left[2 \sin \left(\frac{\theta}{2}+\frac{\pi k}{m}\right)\right]^{d-2}} \\
& =\frac{1}{(2 \pi)^{d-2}} \sum_{k=0}^{m-1}\left[\int_{0}^{\infty} d x \frac{x^{\frac{\theta}{2 \pi}+\frac{k}{m}-1}}{1+x}\right]^{d-2} \\
& =\frac{1}{(2 \pi)^{d-2}} \int_{0}^{\infty} d x_{1} \cdots \int_{0}^{\infty} d x_{d-2}\left(\prod_{i=1}^{d-2} \frac{\left(x_{i}\right)^{\frac{\theta}{2 \pi}}-1}{1+x_{i}}\right)\left[\sum_{k=0}^{m-1}\left(\prod_{i=1}^{d-2} x_{i}\right)^{\frac{k}{m}}\right] \\
& =\frac{1}{(2 \pi)^{d-2}} \int_{0}^{\infty} d x_{1} \cdots \int_{0}^{\infty} d x_{d-2}\left(\prod_{i=1}^{d-2} \frac{\left(x_{i}\right)^{\frac{\theta}{2 \pi}}-1}{1+x_{i}}\right)\left[\frac{1-\prod_{i=1}^{d-2} x_{i}}{1-\left(\prod_{i=1}^{d-2} x_{i}\right)^{\frac{1}{m}}}\right]
\end{aligned}
$$

Then $G_{(n, d)}^{B}(\theta)$ is obtained by replacing $m$ with $\frac{1}{n}$. While this integral expression is valid for all integers $d$, even and odd, for the even integers $d$ it is easier to evaluate the limit (3.16) or use the recurrence relation (3.14) with the $(3+1)$ dimensional result (3.19).

Using the integral (3.24), the two-point function in $d=3$ becomes

$$
G_{(n, 3)}^{B}(\theta)=\frac{1}{2 \pi} \int_{0}^{\infty} \frac{x^{\frac{\theta}{2 \pi}-1}(1-x)}{(1+x)\left(1-x^{n}\right)} d x
$$




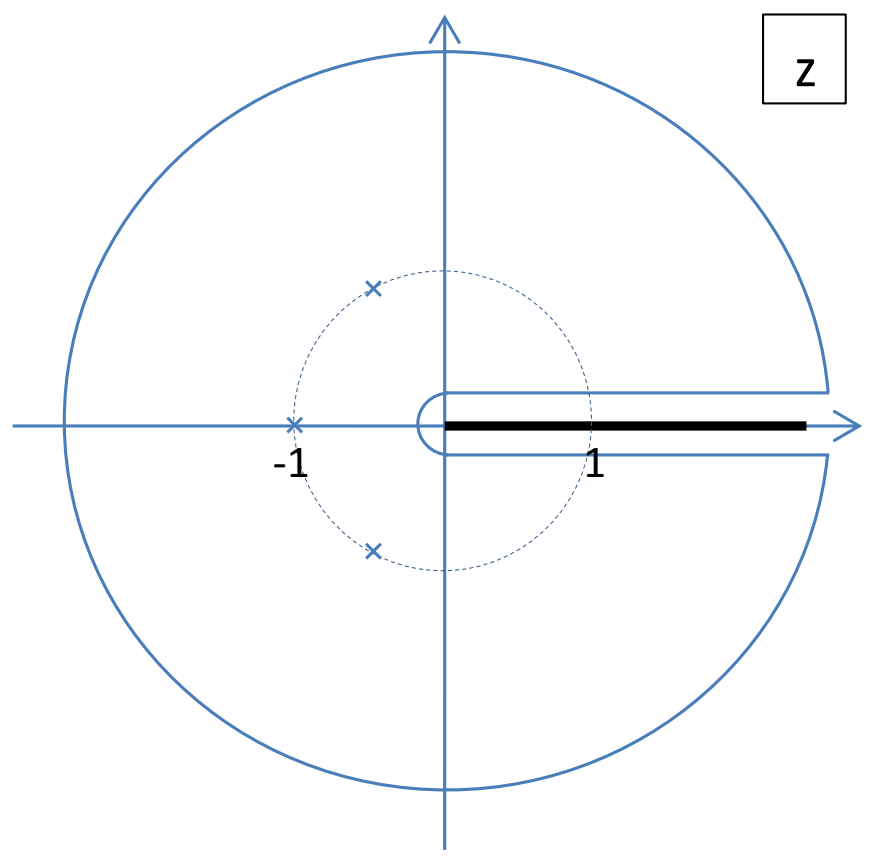

Figure 1. The contour for $d=3$ dimensions and $n=3$

This integral can be done analytically. Essentially it is a contour integral with a branch point at $z=0$ and some poles on the unit circle. For convenience, we can choose a branch cut to be the positive real axis, and a contour shown in figure 1. For an even integer $n$, the poles are

$z=-1$ is a double pole, $z=e^{2 \pi i \frac{\ell}{n}}\left(\ell=1, \cdots, \frac{n}{2}-1, \frac{n}{2}+1, \cdots, n-1\right)$ are simple poles.

For an odd integer $n$, the poles are

$$
z=-1 \text { and } z=e^{2 \pi i \frac{\ell}{n}}(\ell=1, \cdots, n-1) \text { are all simple poles. }
$$

We emphasize that $z=1$ is not a pole. Then for an even integer $n$ :

$$
\begin{aligned}
G_{(n, d)}^{B} & =\frac{1}{2 \pi} \int_{0}^{\infty} \frac{x^{\frac{\theta}{2 \pi}-1}(1-x)}{(1+x)\left(1-x^{n}\right)} d x=\frac{i}{1-e^{2 \pi i\left(\frac{\theta}{2 \pi}-1\right)}} \sum_{\text {Poles }} \operatorname{Res} \\
& =\frac{i}{1-e^{2 \pi i\left(\frac{\theta}{2 \pi}-1\right)}}\left[-(-1)^{\frac{\theta}{2 \pi}}-1\left(\frac{\theta}{n \pi}-1\right)+\sum_{\ell=1, \ell \neq \frac{n}{2}}^{n-1} \frac{e^{2 \pi i \frac{\ell}{n}\left(\frac{\theta}{2 \pi}-1\right)}}{1+e^{2 \pi i \frac{\ell}{n}}}\left(\prod_{j=1, j \neq \ell}^{n-1} \frac{1}{e^{2 \pi i \frac{\ell}{n}}-e^{2 \pi i \frac{j}{n}}}\right)\right] \\
& =\frac{1}{2 \sin \frac{\theta}{2}}\left[1-\frac{\theta}{\pi n}-\frac{i}{n} \sum_{\ell=1, \ell \neq n / 2}^{n-1} e^{i \theta\left(\frac{\ell}{n}-\frac{1}{2}\right)} \tan \frac{\pi \ell}{n}\right]
\end{aligned}
$$


while for an odd integer $n$ :

$$
\begin{aligned}
G_{(n, d)}^{B} & =\frac{1}{2 \pi} \int_{0}^{\infty} \frac{\frac{\theta}{2 \pi}^{\frac{\theta}{2 \pi}}(1-x)}{(1+x)\left(1-x^{n}\right)} d x=\frac{i}{1-e^{2 \pi i\left(\frac{\theta}{2 \pi}-1\right)}} \sum_{\text {Poles }} \operatorname{Res} \\
& =\frac{i}{1-e^{2 \pi i\left(\frac{\theta}{2 \pi}-1\right)}}\left[(-1)^{\frac{\theta}{2 \pi}-1}+\sum_{\ell=1}^{n-1} \frac{e^{2 \pi i \frac{\ell}{n}\left(\frac{\theta}{2 \pi}-1\right)}}{1+e^{2 \pi i \frac{\ell}{n}}}\left(\prod_{j=1, j \neq \ell}^{n-1} \frac{1}{e^{2 \pi i \frac{\ell}{n}}-e^{2 \pi i \frac{j}{n}}}\right)\right] \\
& =\frac{1}{2 \sin \frac{\theta}{2}}\left[1-\frac{i}{n} \sum_{\ell=1}^{n-1} e^{i \theta\left(\frac{\ell}{n}-\frac{1}{2}\right)} \tan \frac{\pi \ell}{n}\right] .
\end{aligned}
$$

Therefore, for $d=3$ dimensions the results for $n=1,2,3$ are eqs. (3.21)-(3.23).

Given the results for $d=3$ dimensions, the two-point functions for $d=5$ dimensions can be obtained by using the recurrence relation (3.14):

$$
\begin{aligned}
G_{(1,5)}^{B}(\theta) & =\frac{1}{\left(2 \sin \frac{\theta}{2}\right)^{3}}, \\
G_{(2,5)}^{B}(\theta) & =\frac{2 \pi-\theta+\sin \theta}{2 \pi\left(2 \sin \frac{\theta}{2}\right)^{3}}, \\
G_{(3,5)}^{B}(\theta) & =\frac{1}{108\left(2 \sin \frac{\theta}{2}\right)^{3}}\left[108-70 \sqrt{3} \sin \left(\frac{\theta}{6}\right)+7 \sqrt{3} \sin \left(\frac{5 \theta}{6}\right)+5 \sqrt{3} \sin \left(\frac{7 \theta}{6}\right)\right] .
\end{aligned}
$$

In appendix $\mathrm{A}$, we also compute the two-point function for $d=5$ dimensions and $n=1,2,3$ by directly evaluating the contour integral

$$
G_{(n, 5)}^{B}(\theta)=\frac{1}{(2 \pi)^{3}} \int_{0}^{\infty} d x \int_{0}^{\infty} d y \int_{0}^{\infty} d z \frac{(x y z)^{\frac{\theta}{2 \pi}-1}(1-x y z)}{(1+x)(1+y)(1+z)\left(1-(x y z)^{n}\right)}
$$

and the results are exactly the same.

\section{Thermal corrections to entanglement entropy}

General results for thermal corrections to entanglement entropy were given in ref. [14]. Here we will verify these general results in arbitrary dimension for the specific case of a conformally coupled scalar. To perform the check, we will use the fact that the $n \rightarrow 1$ limit of the Rényi entropies yields the entanglement entropy.

The Green's function $G_{(n, d)}^{B}(\theta)$ has an expansion near $n=1$ of the form

$$
G_{(n, d)}^{B}(\theta)=G_{(1, d)}^{B}(\theta)+(n-1) \delta G_{(d)}^{B}(\theta)+\mathcal{O}(n-1)^{2} .
$$

From the definition (3.12) and the main result (2.6), we have that

$$
\delta S_{E}=-\frac{\delta G_{(d)}^{B}(2 \theta)}{G_{(1, d)}^{B}(2 \theta)} e^{-\beta E_{\psi}}+o\left(e^{-\beta E_{\psi}}\right) .
$$

Note that $\delta G_{(d)}^{B}(\theta)$ will also satisfy the recurrence relation (3.14). Thus it is enough to figure out the thermal corrections for the smallest dimensions $d=3$ and $d=4$. The result in $d>4$ will then follow from the recurrence. 
Let us check that the expression (4.2) agrees with ref. [14] in the cases $d=3$ and $d=4$. In the case $d=3$, we can evaluate the relevant contour integral (3.25) in the limit $n \rightarrow 1$ :

$$
\begin{aligned}
G_{(n, 3)}^{B}(\theta) & =\frac{1}{2 \pi} \int_{0}^{\infty} \frac{x^{\frac{\theta}{2 \pi}}-1}{1+x} d x+\frac{n-1}{2 \pi} \int_{0}^{\infty} \frac{x^{\frac{\theta}{2 \pi}} \log x}{1-x^{2}}+\mathcal{O}(n-1)^{2} \\
& =\frac{1}{2} \frac{1}{\sin \frac{\theta}{2}}-(n-1) \frac{\pi}{8} \frac{1}{\cos ^{2} \frac{\theta}{4}}+\mathcal{O}(n-1)^{2}
\end{aligned}
$$

From eqs. (4.2) and (4.3), we then have

$$
\delta S_{E}=\frac{\pi}{2} \tan \left(\frac{\theta}{2}\right) e^{-\beta / 2 R}+o\left(e^{-\beta / 2 R}\right) .
$$

For $d=4$, we expand eq. (3.19) near $n=1$ :

$$
G_{(n, 4)}^{B}(\theta)=\frac{1}{4 \sin ^{2} \frac{\theta}{2}}\left(1+(n-1)\left(-2+\theta \cot \frac{\theta}{2}\right)+\mathcal{O}(n-1)^{2}\right) .
$$

We find from eqs. (4.2) and (4.5) that

$$
\delta S_{E}=2(1-\theta \cot \theta) e^{-\beta / R}+o\left(e^{-\beta / R}\right) .
$$

The expressions (4.4) and (4.6) are precisely the results found for the conformally coupled scalar in ref. [14] in $d=3$ and $d=4$ respectively.

Indeed, for general $d$, the result in ref. [14] for the conformally coupled scalar is

$$
\delta S_{E}=\frac{d-2}{2} I_{d-2}(\theta) e^{-\beta(d-2) / 2 R}+o\left(e^{-\beta(d-2) / 2 R}\right) .
$$

where the definition (1.8) of $I_{d}(\theta)$ was given in the introduction. If our result (4.2) for the thermal correction is correct, we can relate $I_{d}(\theta)$ and $\delta G_{(d)}^{B}(\theta)$ :

$$
\delta G_{(d)}^{B}(2 \theta)=-\frac{d-2}{2}(2 \sin \theta)^{2-d} I_{d-2}(\theta),
$$

where we have used the fact that $G_{(1, d)}^{B}(2 \theta)=(2 \sin \theta)^{2-d}$.

To check that our thermal corrections are correct for general $d$, we will use a roundabout method. In ref. [14], it was also found that the function $I_{d}(\theta)$ satisfies a recurrence relation

$$
I_{d}(\theta)-I_{d-2}(\theta)=-2 \pi \frac{\operatorname{Vol}\left(S^{d-2}\right)}{\operatorname{Vol}\left(S^{d-1}\right)} \frac{\sin ^{d-2} \theta}{(d-1)(d-2)} .
$$

We will use our recurrence relation (3.14) and the tentative identification (4.8) to replace $I_{d}(\theta)$ with $I_{d-2}(\theta)$ in the above expression:

$$
\begin{aligned}
I_{d}(\theta) & =-\frac{2}{d}(2 \sin \theta)^{d} \delta G_{(d+2)}^{B}(2 \theta) \\
& =-\frac{2(2 \sin \theta)^{d}}{d(d-1)(d-2)}\left[\left(\frac{d-2}{2}\right)^{2}+\frac{1}{4} \frac{\partial^{2}}{\partial \theta^{2}}\right] \delta G_{(d)}^{B}(2 \theta) \\
& =\frac{(2 \sin \theta)^{d}}{4 d(d-1)}\left[(d-2)^{2}+\frac{\partial^{2}}{\partial \theta^{2}}\right](2 \sin \theta)^{2-d} I_{d-2}(\theta) .
\end{aligned}
$$

Then we have checked that the resulting differential equation in $I_{d-2}(\theta)$ is solved by the integral formula (1.8). 


\section{Numerical check}

We check numerically the thermal Rényi entropy corrections obtained in section 3.1. The algorithm we use was described in detail in ref. [14], so we shall be brief. (The method is essentially that of ref. [2].) The action for a conformally coupled scalar on $\mathbb{R} \times S^{d-1}$ is

$$
S=-\frac{1}{2} \int d^{d} x \sqrt{-g}\left[\left(\partial_{\mu} \phi\right)\left(\partial^{\mu} \phi\right)+\xi \mathcal{R} \phi^{2}\right],
$$

where $\xi$ is the conformal coupling $\xi=(d-2) / 4(d-1)$ and $\mathcal{R}$ is the Ricci scalar curvature. Given that the region $A$ can be characterized by the polar angle $\theta$ on $S^{d-1}$, we write the Hamiltonian as a sum $H=\sum_{\vec{l}} H_{\vec{l}}$, where we have replaced all the other angles on $S^{d-1}$ by corresponding angular momentum quantum numbers $\left|l_{1}\right| \leq l_{2} \leq \cdots \leq l_{d-2} \equiv m$. The individual Hamiltonians take the form

$$
H_{\vec{l}}=\frac{1}{2 R^{2}} \int_{0}^{\pi}\left\{R^{2} \Pi_{\vec{l}}^{2}-\Phi_{\vec{l}} \partial_{\theta}^{2} \Phi_{\vec{l}}+\frac{1}{4}(2 m+d-2)(2 m+d-4) \frac{\Phi_{\vec{l}}^{2}}{\sin ^{2} \theta}\right\} d \theta .
$$

It is convenient to discretize $H_{\vec{l}}$. In $d \geq 4$, we introduce a lattice in $\theta$, while in $d=3$, a lattice in $\cos \theta$ appears to work better. The entanglement and Rényi entropies can then be expressed in terms of two-point functions restricted to the region $A$. In particular, the Rényi entropy can be expressed as

$$
S_{n}(T)=S_{n}(0)+\sum_{m=1}^{\infty} \operatorname{dim}(m) S_{n}^{(m)}
$$

where

$$
\operatorname{dim}(m)=\left(\begin{array}{c}
d+m-2 \\
d-2
\end{array}\right)-\left(\begin{array}{c}
d+m-4 \\
d-2
\end{array}\right)
$$

and

$$
S_{n}^{(m)}=\frac{1}{1-n} \log \operatorname{tr}\left[\left(C_{m}+\frac{1}{2}\right)^{n}-\left(C_{m}-\frac{1}{2}\right)^{n}\right] .
$$

The matrix $C_{m}\left(\theta_{1}, \theta_{2}\right)$ has a continuum version

$$
C_{m}\left(\theta_{1}, \theta_{2}\right)^{2}=\int_{0}^{\theta_{0}} d \theta\left\langle\Phi_{\vec{l}}\left(\theta_{1}\right) \Phi_{\vec{l}}(\theta)\right\rangle\left\langle\Pi_{\vec{l}}(\theta) \Pi_{\vec{l}}\left(\theta_{2}\right)\right\rangle .
$$

The thermal two-point functions have the following expressions:

$$
\begin{aligned}
& \left\langle\Phi_{\vec{l}}(\theta) \Phi_{\bar{l}^{-}}\left(\theta^{\prime}\right)\right\rangle=\frac{1}{2} \sum_{l=m}^{\infty} U_{l}(\theta) \frac{1}{\omega_{l}} \operatorname{coth} \frac{\omega_{l}}{2 T} U_{l}\left(\theta^{\prime}\right), \\
& \left\langle\Pi_{\vec{l}}(\theta) \Pi_{\bar{l}}\left(\theta^{\prime}\right)\right\rangle=\frac{1}{2} \sum_{l=m}^{\infty} U_{l}(\theta) \omega_{l} \operatorname{coth} \frac{\omega_{l}}{2 T} U_{l}\left(\theta^{\prime}\right),
\end{aligned}
$$

where $\omega_{l} \equiv \frac{1}{R}\left(l+\frac{d-2}{2}\right)$. In the continuum limit, the matrix $U_{l}(\theta)$ is an orthogonal transformation involving associated Legendre functions whose explicit form is given in ref. [14]. In practice, we use the discretized version of $U_{l}(\theta)$ that follows from the discretized $H_{\vec{l}}$. 


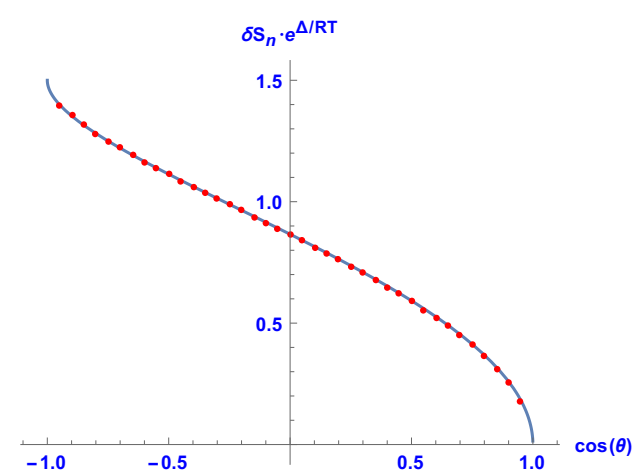

Figure 2. $\delta S_{n=3}$ in $(2+1) \mathrm{D}, 400$ grid points.

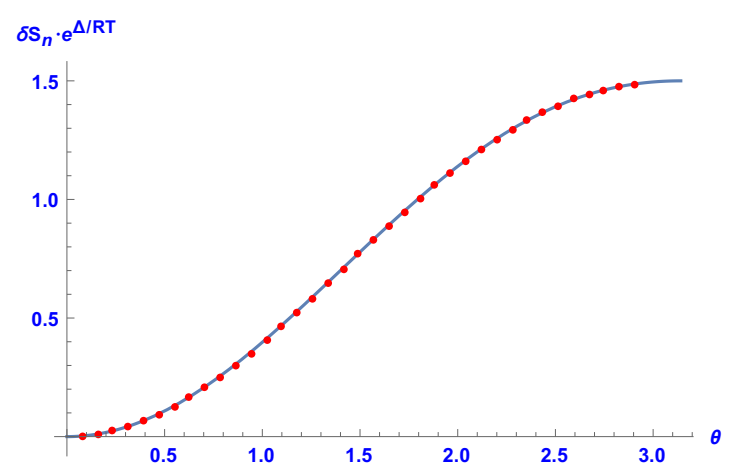

Figure 3. $\delta S_{n=3}$ in $(3+1)$ D, 400 grid points.

As discussed in ref. [7, 14], if the limit $\theta_{0} \rightarrow \pi$ is taken first, the leading correction to $\delta S_{n}$ comes from the thermal Rényi entropy instead of from the entanglement:

$$
\delta S_{n}=\left[-g \frac{n}{1-n}+\mathcal{O}\left(1-\frac{\theta_{0}}{\pi}\right)^{2 \Delta}\right] e^{-\Delta / R T}+o\left(e^{-\Delta / R T}\right) .
$$

Indeed, when $\pi-\theta$ is small compared to $R T$, the Rényi entropy looks like the thermal Rényi entropy and approaches it in the limit $\theta \rightarrow \pi$. To isolate the $e^{-\Delta / R T}$ dependence of $\delta S_{n}$ analytically, we can expand the coth-function in the thermal two-point functions (5.6) and (5.7). In principle, one can evaluate eq. (5.3) to obtain $\delta S_{n}(T)$. Since we are interested in the low temperature limit, the contributions from $S_{n}^{(m)}(m>0)$ to $\delta S_{n}(T)$ are exponentially suppressed compared with $S_{n}^{(0)}$. Therefore, in the limit of small $T$, we obtain the expansion of eq. (5.3):

$$
\delta S_{n}=\frac{n}{2(n-1)} \operatorname{tr}\left[\delta C_{0} \cdot C_{0}^{-1} \cdot \frac{\left(C_{0}+\frac{1}{2}\right)^{n-1}-\left(C_{0}-\frac{1}{2}\right)^{n-1}}{\left(C_{0}+\frac{1}{2}\right)^{n}-\left(C_{0}-\frac{1}{2}\right)^{n}}\right] e^{-\omega_{0} / T}+\cdots,
$$

where

$$
\begin{aligned}
\delta C_{m}\left(\theta_{1}, \theta_{2}\right) & \equiv \int_{0}^{\theta_{0}} d \theta\left[\left\langle\Phi_{\vec{l}}\left(\theta_{1}\right) \Phi_{\vec{l}}(\theta)\right\rangle \delta \Pi_{m}\left(\theta, \theta_{2}\right)+\delta \Phi_{m}\left(\theta_{1}, \theta\right)\left\langle\Pi_{\vec{l}}(\theta) \Pi_{\vec{l}}\left(\theta_{2}\right)\right\rangle\right], \\
\delta \Phi_{m}\left(\theta, \theta^{\prime}\right) & \equiv U_{m}(\theta) \frac{1}{\omega_{m}} U_{m}\left(\theta^{\prime}\right), \\
\delta \Pi_{m}\left(\theta, \theta^{\prime}\right) & \equiv U_{m}(\theta) \omega_{m} U_{m}\left(\theta^{\prime}\right) .
\end{aligned}
$$

Some results of $\delta S_{n}$ in different dimensions are shown in figures 2-5. To diagonalize the matrices with enough accuracy, high precision arithmetic is required.

\section{Discussion}

Our main result provides a way to calculate the leading thermal correction to a specific kind of Rényi entropy for a CFT. In particular, the CFT should live on $\mathbb{R} \times S^{d-1}$, and the region is a cap on the sphere with opening angle $2 \theta$. We demonstrated that this correction 


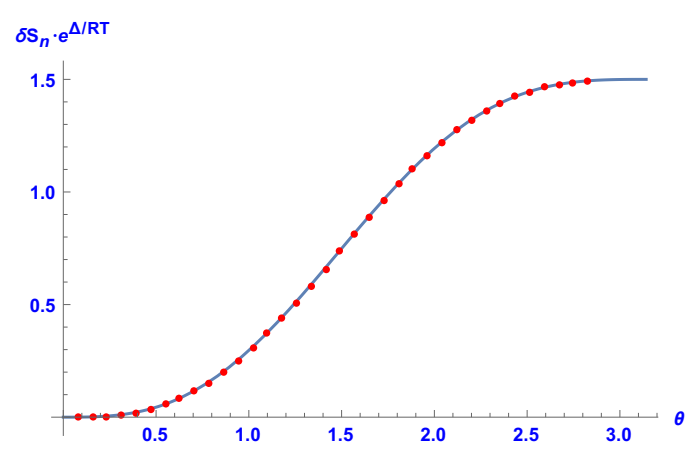

Figure 4. $\delta S_{n=3}$ in $(4+1) \mathrm{D}, 400$ grid points.

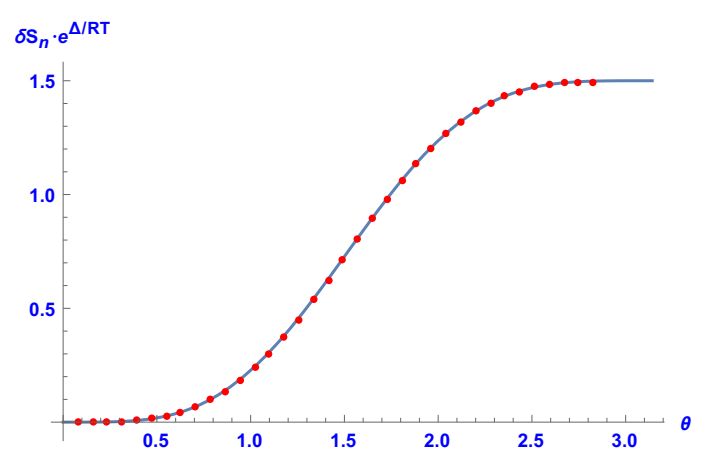

Figure 5. $\delta S_{n=3}$ in $(5+1) \mathrm{D}, 400$ grid points.

is equivalent to knowing the two-point function on a certain conical space of the operator that creates the first excited state. In the case of a conformally coupled free scalar, the scalar field itself creates the first excited state, and the two point function can be computed by the method of images. In the $n \rightarrow 1$ limit, Rényi entropy becomes entanglement entropy, and we were able to show that our results agree with ref. [14]. We were also able to check our thermal corrections for $n>1$ numerically, using a method based on ref. [2].

We would like to make two observations about our results. The first is that our thermal Rényi entropy corrections are often but not always invariant under the replacement $\theta \rightarrow 2 \pi n-\theta$. (The exceptions are $\delta S_{n}$ for even $n$ and odd $d$.) A similar observation was made in ref. [7] in the $1+1$ dimensional case. There, the invariance could be explained by moving twist operators around the torus (or cylinder). The branch cut joining two twist operators is the same cut along which the different copies of the torus are glued together. By moving a twist operator $n$ times around the torus, $n$ branch cuts are equivalent to nothing while $n-1$ branch cuts are equivalent to a single branch cut that moves one down a sheet rather than up a sheet. Perhaps in higher dimensions the invariance can be explained in terms of surface operators that glue the $n$ copies of $S^{1} \times S^{d-1}$ together. It is not clear to us how to generalize the argument. It is tempting to speculate that the invariance is spoiled in odd dimensions (even dimensional spheres) because only odd dimensional spheres are Hopf fibrations over projective space.

The second observation is that the leading corrections to $\delta S_{n}$ for small caps $\theta \ll 1$ have a power series expansion that starts with the terms $a \theta^{d-2}+b \theta^{d}+\ldots$. In $1+1$ dimensions, the power series starts with $\theta^{2}[7]$. When we bring two twist operators together, the twist operators can be replaced by their operator product expansion, a leading term of which is the stress tensor. The $\theta^{2}$ term in $\delta S_{n}$ comes from a three point function of the stress tensor with the operators that create and annihilate the first excited state. The two in the exponent of $\theta^{2}$ comes from the scaling dimension of the stress tensor, and the coefficient of the $\theta^{2}$ can be related to the scaling dimension of the twist operators [7]. In our higher dimensional case, we can replace the surface operator along the boundary of the cap by an operator product expansion at a point. Because of Wick's theorem, the leading operator that can contribute to $\delta S_{n}$ will be $\phi^{2}$ which has dimension $d-2$. The subleading $\theta^{d}$ term may come from the stress tensor and descendants of $\phi^{2}$. A more detailed analysis might 
shed some light on the structure of these surface operators. ${ }^{3}$

In addition to developing the above observations, we give a couple of projects for future research. One would be to compute these thermal corrections for free fermions. The two point function on this conical space can quite likely be computed. It would be interesting to see how the results compare to the scalar. Given the importance of boundary terms for the scalar, it would also be nice to get further confirmation of the general story for thermal corrections to entanglement entropy presented in ref. [14].

Another interesting project would be to see how to obtain these results holographically. As the corrections are subleading in a large central charge (or equivalently large $N$ ) expansion, they would not be captured by the Ryu-Takayanagi formula [21]. However, it may be possible to generalize the computation in $d=2[11]$ to $d>2$. Finally, it would be interesting to see what can be said about negativity in higher dimensions. See refs. [22, 23] for the two dimensional case.

\section{Acknowledgments}

We would like to thank Michael Spillane, Pin-Ju Tien, and Ricardo Vaz for useful discussions. C. H. and J. N. were supported in part by the National Science Foundation under Grant No. PHY13-16617. C. H. thanks the Sloan Foundation for partial support.

\section{A Two-point functions in $d=(4+1)$ dimensions}

In this appendix, we compute the two-point function for $d=(4+1)$ dimensions given by eq. (3.31). In contrast to eq. (3.25), eq. (3.31) is a multi-variable contour integral and we need to do some changes of variables first. The procedure used here can be applied in higher dimensions and for any integer $n \geq 1$. In $d=5$, we find

$$
\begin{aligned}
G_{(n, 5)}^{B}(\theta) & =\frac{1}{(2 \pi)^{3}} \int_{0}^{\infty} d x \int_{0}^{\infty} d y \int_{0}^{\infty} d z \frac{(x y z)^{\frac{\theta}{2 \pi}}-1(1-x y z)}{(1+x)(1+y)(1+z)\left(1-(x y z)^{n}\right)} \\
& =\frac{1}{(2 \pi)^{3}} \int_{0}^{\infty} d x \int_{0}^{\infty} d y \int_{0}^{\infty} \frac{1}{x y} d z^{\prime} \frac{z^{\prime \frac{\theta}{2 \pi}-1}\left(1-z^{\prime}\right)}{(1+x)(1+y)\left(1+\frac{z^{\prime}}{x y}\right)\left(1-z^{\prime n}\right)} \\
& =\frac{1}{(2 \pi)^{3}} \int_{0}^{\infty} d x \int_{0}^{\infty} d y \int_{0}^{\infty} d z^{\prime} \frac{z^{\prime \frac{\theta}{2 \pi}-1}\left(1-z^{\prime}\right)}{(1+x)(1+y)\left(x y+z^{\prime}\right)\left(1-z^{\prime n}\right)} \\
& =\frac{1}{(2 \pi)^{3}} \int_{0}^{\infty} d x \int_{0}^{\infty} \frac{1}{x} d y^{\prime} \int_{0}^{\infty} d z^{\prime} \frac{z^{\prime} \frac{\theta}{2 \pi}-1\left(1-z^{\prime}\right)}{(1+x)\left(1+\frac{y^{\prime}}{x}\right)\left(y^{\prime}+z^{\prime}\right)\left(1-z^{\prime n}\right)} \\
& =\frac{1}{(2 \pi)^{3}} \int_{0}^{\infty} d x \int_{0}^{\infty} d y^{\prime} \int_{0}^{\infty} d z^{\prime} \frac{z^{\prime \frac{\theta}{2 \pi}}-1\left(1-z^{\prime}\right)}{(1+x)\left(x+y^{\prime}\right)\left(y^{\prime}+z^{\prime}\right)\left(1-z^{\prime n}\right)} \\
& =\frac{1}{(2 \pi)^{3}} \int_{0}^{\infty} d x \int_{0}^{\infty} d y \int_{0}^{\infty} d z \frac{z^{\frac{\theta}{2 \pi}}-1(1-z)}{(1+x)(x+y)(y+z)\left(1-z^{n}\right)},
\end{aligned}
$$

where

$$
z^{\prime} \equiv x y z, \quad y^{\prime} \equiv x y,
$$

\footnotetext{
${ }^{3}$ See refs. [16, 18-20] for related work on higher dimensional analogs of twist operators.
} 
and we drop the ' in the last line. Performing the integration over $x$ and $y$ in eq. (A.1), we obtain

$$
G_{(n, 5)}^{B}(\theta)=\frac{1}{2(2 \pi)^{3}} \int_{0}^{\infty} d z \frac{z\left(\pi^{2}+(\log z)^{2}\right)(z-1)}{(1+z)\left(z^{n}-1\right)} .
$$

This integral can be done analytically by choosing the same branch cut and contour used in the $d=(2+1)$ dimensional case discussed in section 3.2 ; the poles are exactly the same. The result for $n=1$ is eq. (3.28). For $n=2$ the result is eq. (3.29). To obtain this result, one needs the following intermediate results

$$
\begin{aligned}
\int_{0}^{\infty} d z \frac{z(z-1)}{(1+z)\left(z^{2}-1\right)} & =\frac{\pi\left(\frac{\theta}{2 \pi}-1\right)}{\sin \left[\pi\left(\frac{\theta}{2 \pi}-1\right)\right]} \\
\int_{0}^{\infty} d z \frac{z \log z(z-1)}{(1+z)\left(z^{2}-1\right)} & =\frac{\pi\left[1-\pi\left(\frac{\theta}{2 \pi}-1\right) \cot \left(\pi\left(\frac{\theta}{2 \pi}-1\right)\right)\right]}{\sin \left(\pi\left(\frac{\theta}{2 \pi}-1\right)\right)} .
\end{aligned}
$$

Similarly, for $n=3$ one can follow exactly the same procedure and find eq. (3.30). Again, one needs some intermediate steps:

$$
\begin{gathered}
\int_{0}^{\infty} d z \frac{z(z-1)}{(1+z)\left(z^{3}-1\right)}=\frac{\pi\left[\sqrt{3} \cos \left(\frac{\pi}{6}\left(\frac{\theta}{2 \pi}-1\right)\right)-3 \sin \left(\frac{\pi}{6}\left(\frac{\theta}{2 \pi}-1\right)\right)\right]}{3\left[\cos \left(\frac{\pi}{6}\left(\frac{\theta}{2 \pi}-1\right)\right)+\cos \left(\frac{\pi}{2}\left(\frac{\theta}{2 \pi}-1\right)\right)+\cos \left(\frac{5 \pi}{6}\left(\frac{\theta}{2 \pi}-1\right)\right)\right]} \\
\int_{0}^{\infty} d z \frac{z \log z(z-1)}{(1+z)\left(z^{3}-1\right)}=\frac{\pi^{2}}{18\left[\cos \left(\frac{1}{6} \pi\left(\frac{\theta}{2 \pi}-1\right)\right)+\cos \left(\frac{1}{2} \pi\left(\frac{\theta}{2 \pi}-1\right)\right)+\cos \left(\frac{5}{6} \pi\left(\frac{\theta}{2 \pi}-1\right)\right)\right]^{2}} \\
. \quad\left[-6 \cos \left(\frac{1}{3} \pi\left(\frac{\theta}{2 \pi}-1\right)\right)-6 \cos \left(\frac{2}{3} \pi\left(\frac{\theta}{2 \pi}-1\right)\right)+6 \cos \left(\pi\left(\frac{\theta}{2 \pi}-1\right)\right)\right. \\
\left.+2 \sqrt{3} \sin \left(\frac{1}{3} \pi\left(\frac{\theta}{2 \pi}-1\right)\right)+4 \sqrt{3} \sin \left(\frac{2}{3} \pi\left(\frac{\theta}{2 \pi}-1\right)\right)+2 \sqrt{3} \sin \left(\pi\left(\frac{\theta}{2 \pi}-1\right)\right)-3\right]
\end{gathered}
$$

\section{B Examples of thermal corrections to Rényi entropies}

In this appendix we summarize the thermal corrections to the $n$th Rényi entropy for the conformally coupled scalar. The Rényi entropy is calculated with respect to a cap of opening angle $2 \theta$ on $S^{d-1}$ for small values of $d$ and $n$. Define the coefficient $f(\theta)$ such that $\delta S_{n}=S_{n}(T)-S_{n}(0)$ has the form

$$
\delta S_{n}=f(\theta) e^{-\beta \Delta / R}+o\left(e^{-\beta \Delta / R}\right),
$$

where $\Delta=\frac{d-2}{2}$ is the scaling dimension of the free scalar and $R$ is the radius of $S^{d-1}$. The following tables give the form of $f(\theta)$. (We also give results for the entanglement entropy, denoted EE.) For $(2+1)$ dimensions:

$$
\begin{array}{c|c}
\mathrm{EE} & \frac{\pi}{2} \tan \left(\frac{\theta}{2}\right) \\
\hline n=2 & \frac{2 \theta}{\pi} \\
\hline n=3 & \sqrt{3} \sin \left(\frac{\theta}{3}\right)
\end{array}
$$


For $(3+1)$ dimensions:

$$
\begin{array}{c|c}
\mathrm{EE} & 2-2 \theta \cot (\theta) \\
\hline n=2 & 1-\cos (\theta) \\
\hline n=3 & \frac{4}{3}\left[2+\cos \left(\frac{2 \theta}{3}\right)\right] \sin ^{2}\left(\frac{\theta}{3}\right)
\end{array}
$$

For $(4+1)$ dimensions:

\begin{tabular}{c|c}
$\mathrm{EE}$ & $3 \pi \csc (\theta) \sin ^{4}\left(\frac{\theta}{2}\right)$ \\
\hline$n=2$ & $\frac{1}{\pi}[2 \theta-\sin (2 \theta)]$ \\
\hline$n=3$ & $\frac{1}{6 \sqrt{3}}\left[51+44 \cos \left(\frac{2 \theta}{3}\right)+10 \cos \left(\frac{4 \theta}{3}\right)\right] \sin ^{3}\left(\frac{\theta}{3}\right)$
\end{tabular}

For $(5+1)$ dimensions:

$$
\begin{array}{c|c}
\mathrm{EE} & \frac{2}{3}[5+\cos (2 \theta)-6 \theta \cot (\theta)] \\
\hline n=2 & 2[2+\cos (\theta)] \sin ^{4}\left(\frac{\theta}{2}\right) \\
\hline n=3 & \frac{16}{81}\left[50+60 \cos \left(\frac{2 \theta}{3}\right)+21 \cos \left(\frac{4 \theta}{3}\right)+4 \cos (2 \theta)\right] \sin ^{4}\left(\frac{\theta}{3}\right)
\end{array}
$$

Open Access. This article is distributed under the terms of the Creative Commons Attribution License (CC-BY 4.0), which permits any use, distribution and reproduction in any medium, provided the original author(s) and source are credited.

\section{References}

[1] L. Bombelli, R.K. Koul, J. Lee and R.D. Sorkin, A Quantum Source of Entropy for Black Holes, Phys. Rev. D 34 (1986) 373 [INSPIRE].

[2] M. Srednicki, Entropy and area, Phys. Rev. Lett. 71 (1993) 666 [hep-th/9303048] [INSPIRE].

[3] H. Casini and M. Huerta, A c-theorem for the entanglement entropy, J. Phys. A 40 (2007) 7031 [cond-mat/0610375] [INSPIRE].

[4] H. Casini and M. Huerta, On the RG running of the entanglement entropy of a circle, Phys. Rev. D 85 (2012) 125016 [arXiv:1202.5650] [INSPIRE].

[5] T.J. Osborne and M.A. Nielsen, Entanglement in a simple quantum phase transition Phys. Rev. A 66 (2002) 032110 [quant-ph/0202162].

[6] G. Vidal, J.I. Latorre, E. Rico and A. Kitaev, Entanglement in quantum critical phenomena, Phys. Rev. Lett. 90 (2003) 227902 [quant-ph/0211074] [INSPIRE].

[7] J. Cardy and C.P. Herzog, Universal Thermal Corrections to Single Interval Entanglement Entropy for Two Dimensional Conformal Field Theories, Phys. Rev. Lett. 112 (2014) 171603 [arXiv: 1403.0578] [INSPIRE].

[8] C.P. Herzog and M. Spillane, Tracing Through Scalar Entanglement, Phys. Rev. D 87 (2013) 025012 [arXiv: 1209.6368] [INSPIRE].

[9] T. Azeyanagi, T. Nishioka and T. Takayanagi, Near Extremal Black Hole Entropy as Entanglement Entropy via $A d S_{2} / C F T_{1}$, Phys. Rev. D 77 (2008) 064005 [arXiv:0710.2956] [INSPIRE].

[10] C.P. Herzog and T. Nishioka, Entanglement Entropy of a Massive Fermion on a Torus, JHEP 03 (2013) 077 [arXiv: 1301.0336] [INSPIRE]. 
[11] T. Barrella, X. Dong, S.A. Hartnoll and V.L. Martin, Holographic entanglement beyond classical gravity, JHEP 09 (2013) 109 [arXiv: 1306.4682] [INSPIRE].

[12] S. Datta and J.R. David, Rényi entropies of free bosons on the torus and holography, JHEP 04 (2014) 081 [arXiv:1311.1218] [inSPIRE].

[13] B. Chen and J.-q. Wu, Single interval Renyi entropy at low temperature, JHEP 08 (2014) 032 [arXiv: 1405.6254] [INSPIRE].

[14] C.P. Herzog, Universal Thermal Corrections to Entanglement Entropy for Conformal Field Theories on Spheres, JHEP 1410 (2014) 28 [arXiv:1407.1358] [INSPIRE].

[15] C. Herzog and M. Spillane, to appear.

[16] J. Cardy, Some results on the mutual information of disjoint regions in higher dimensions, J. Phys. A 46 (2013) 285402 [arXiv:1304.7985] [INSPIRE].

[17] P. Candelas and J.S. Dowker, Field Theories on Conformally Related Space-Times: Some Global Considerations, Phys. Rev. D 19 (1979) 2902 [InSPIRE].

[18] N. Shiba, Entanglement Entropy of Two Spheres, JHEP 07 (2012) 100 [arXiv:1201.4865] [INSPIRE].

[19] L.-Y. Hung, R.C. Myers and M. Smolkin, Twist operators in higher dimensions, JHEP 10 (2014) 178 [arXiv:1407.6429] [INSPIRE].

[20] H. Casini and M. Huerta, Remarks on the entanglement entropy for disconnected regions, JHEP 03 (2009) 048 [arXiv:0812.1773] [INSPIRE].

[21] S. Ryu and T. Takayanagi, Holographic derivation of entanglement entropy from AdS/CFT, Phys. Rev. Lett. 96 (2006) 181602 [hep-th/0603001] [INSPIRE].

[22] V. Eisler and Z. Zimborás, Entanglement negativity in the harmonic chain out of equilibrium, New J. Phys. 16 (2014) 123020.

[23] P. Calabrese, J. Cardy and E. Tonni, Finite temperature entanglement negativity in conformal field theory, J. Phys. A 48 (2015) 015006 [arXiv: 1408.3043] [InSPIRE]. 